\title{
Poster Abstract: BESI: Behavior Learning and Tracking with Wearable and In-Home Sensors - A Dementia Case-Study
}

\author{
Ridwan Alam \\ University of Virginia \\ Charlottesville, VA \\ ridwan@virginia.edu
}

\author{
Nutta Homdee \\ University of Virginia \\ Charlottesville, VA \\ nh4ar@virginia.edu
}

\author{
Sean Wolfe \\ University of Virginia \\ Charlottesville, VA \\ scw2tt@virginia.edu
}

\author{
James Hayes \\ University of Virginia \\ Charlottesville, VA \\ jah4yq@virginia.edu
}

\author{
John Lach \\ University of Virginia \\ Charlottesville, VA \\ jlach@virginia.edu
}

\begin{abstract}
Sensing driven behavior modeling is vital in health applications. Recent advances in machine learning and sensing technologies accelerate such efforts. While wearables facilitate continuous sensing, they lack the computational resources for on-board heavy-weight signal processing and model-based prediction. Moreover, continuous transmission to a remote server drains much energy to achieve reasonable battery life for practical use. The BESI (Behavioral and Environmental Sensing and Intervention) system addresses these challenges to achieve continuous and real-time prediction-based tracking of human behavior. It employs a network of embedded nodes to ensure continuous connection with the wearables, and distributes the feature extraction and the model prediction tasks among these nodes and a local server to achieve real-time performance. In a dementia case-study, the BESI system is used for tracking agitated behavior in patients. It has been deployed in 12 residences of dementia patients, each for 30 days; and is planned for 10 more 60 -day deployments. The system operation, behavior modeling method, and some preliminary result on tracking performance are presented here along with a discussion on future plan for platform optimization and model performance improvement.
\end{abstract}

\section{CCS CONCEPTS}

- Computer systems organization $\rightarrow$ Sensors and actuators.

\section{KEYWORDS}

Wearable, Behavior Models, Deployment, Health Application

\section{ACM Reference Format:}

Ridwan Alam, Nutta Homdee, Sean Wolfe, James Hayes, and John Lach. 2019. Poster Abstract: BESI: Behavior Learning and Tracking with Wearable and In-Home Sensors - A Dementia Case-Study. In International Conference on Internet-of-Things Design and Implementation (IoTDI '19), April 15-18, 2019, Montreal, QC, Canada. ACM, New York, NY, USA, 2 pages. https: //doi.org/10.1145/3302505.3312595

Permission to make digital or hard copies of part or all of this work for personal or classroom use is granted without fee provided that copies are not made or distributed for profit or commercial advantage and that copies bear this notice and the full citation on the first page. Copyrights for third-party components of this work must be honored

For all other uses, contact the owner/author(s).

IoTDI '19, April 15-18, 2019, Montreal, QC, Canada

(c) 2019 Copyright held by the owner/author(s).

ACM ISBN 978-1-4503-6283-2/19/04.

https://doi.org/10.1145/3302505.3312595

\section{INTRODUCTION}

Health applications such as elderly care or outpatient monitoring require continuous behavior tracking. State-of-the-art approaches are mostly focused on activity recognition and confined to controlled settings. Real-world settings bring in large amount of data with various complexities and uncertainties. Practical challenges include ensuring continuous coverage and real-time behavior tracking while reducing the compliance burden. We address this challenge by implementing the BESI system [2], and demonstrate its application in a study on dementia patients. The objective of this study is to prevent agitation escalation. More than $90 \%$ of dementia patients demonstrate agitated behavioral symptoms. To prevent escalation to a health hazard scenario, real-time tracking is critical. We deploy the BESI system in the patients residences, build the behavioral model [1] offline using data from the first half of the deployment, and plug-in the model in the system for real-time tracking for the rest of the deployment. The preliminary results from this on-going study are presented here.

\section{SYSTEM OPERATION}

The BESI system [2] consists of heterogeneous sensing and computing components: wearables (smart watch), mobiles (smart phone,

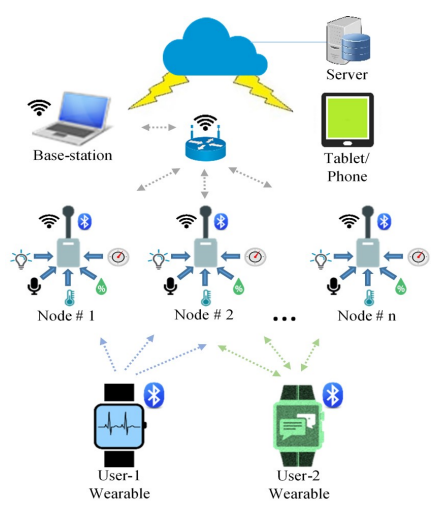

(a) System architecture

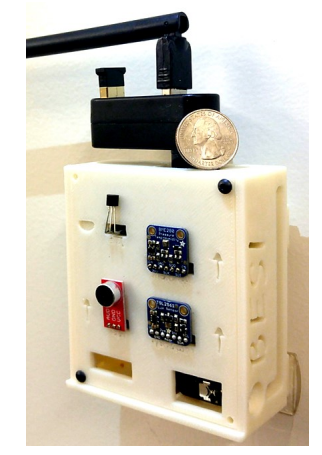

(b) A room-level node
Figure 1: The BESI system comprises of smart watches and phones, embedded nodes, a base-station, and a cloud server. 
tablet), room-level embedded nodes, base-station or local server, and cloud server. The operational relationship of these devices in the architecture are depicted in Fig 1(a). Wearables are used for sensing behavioral cues from motion and physiological signals. These signals are transmitted via Bluetooth to a connected embedded node. The room-level nodes are clients in the distributed network, spread across the residence, and perform a spectrum of sensing and processing tasks, as shown in Fig. 1(b). These nodes are programmed to automatically connect to and seamlessly handover the wearables, thus ensuring continuous coverage. Each node process the received signals and extract representative features, while the base-station manager coordinates these task assignments. The behavior model is hosted on the base-station and uses the features from the nodes for real-time prediction. The modeling pipeline is implemented with the models being an exchangeable module, which allows us to update the model at any point of the deployments. The cloud server facilitates both data backup and remote monitoring of the system performance as well as the behavior model outcomes and trends. The system has been tested for robustness and reliability over the course of the last 10 deployments, each for 30 days, at the residences of dementia patients.

\section{LEARNING AND TRACKING}

The features are extracted from the wearable motion data on each embedded node independently. Every 30 seconds, a one-minute window of the 3-axes accelerometer signal from the wearable is used to extract one feature instance (vector) for that time index. The elements of the feature instance are from three categories: statistical, frequency, and energy features. The statistical features comprise mean, maximum, variance, median, inter-quartile range, and cross-correlation of the three axes. The frequency features are mean crossing rate and the power spectrum density in three frequency ranges. And, the energy features are mean and variance of the Teager energy in that one-minute window. We found these features representative of the physical manifestation of agitated behaviors of dementia patients [1].

The base-station task manager collects and synchronizes the feature instances from the distributed nodes. During the learning phase (first half of the deployment), these features are labeled using the surveys provided by the users. In the dementia study, the caregivers provide the time and intensity of the agitation episodes. During the tracking phase, the feature instances are directly fed into the model to acquire a prediction on the behavior. In this phase, we acquire ground truth by prompting the user for feedback on the prediction. For dementia caregivers, we are currently working on generating adaptive prompts depending on the context and confidence of the prediction.

\section{PRELIMINARY EVALUATION}

We evaluate the performance of multiple standard models such as support vector machines (SVM), adaptive boosting (AdaBoost), and ensemble of decision trees by bagging (Tree-Bagging). These models are trained and validated on the data from the learning phase, and the outcome of the 10-fold cross validation is shown in Fig. 2(a), which demonstrates the similarity in learning performance by these models. These models are used during the tracking phase to evaluate

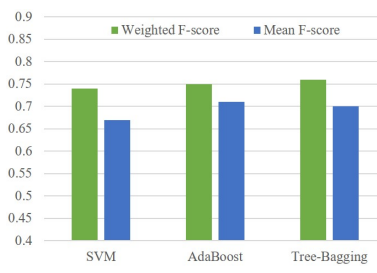

(a) Learning

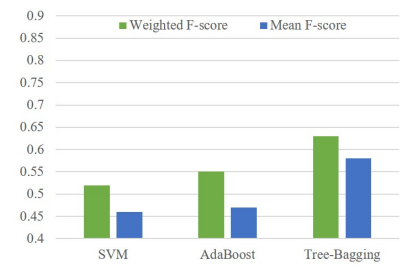

(b) Tracking
Figure 2: Comparison of model performances for learning and tracking agitated behavior in dementia.

their performance on unknown data, as shown in Fig. 2(b). The ensemble by bagging appears to be more robust compared to other models. We use average and weighted F-score as evaluation metrics, rather than accuracy, as the data are highly populated with negative samples (non-agitation periods). For the same reason, the mean F-scores appear lower than the weighted scores. Also, we notice that the lower performance of the models are mostly attributed to the large number of false-positives (predicting agitation when it's not happening) rather than too many false-negatives (missing to detect an agitation). This may be attributed to the contextual and inter-personal variations among the patterns of agitation, which emphasizes the need for personalized and context-aware behavior models for such applications.

\section{CONCLUSION}

In this work, we have successfully completed the implementation of a robust and reliable heterogeneous sensing platform. We are currently halfway in our deployments, and we are improving both the system and the behavior models iteratively along these deployments. Future improvements to the system are focused on generalization by incorporating more commercial IoT devices even with different communication protocols and to evaluate the efficiency of the user feedback loop for providing just-in-time adaptive interventions. For the behavior modeling pipeline, we are working on incorporating context-awareness from other sensing modalities (e.g. audio noise) into the models. Also, we are exploring various personalization techniques along with existing transfer learning approaches to acquire better performance in learning and tracking human behavior.

\section{ACKNOWLEDGMENTS}

To our collaborators from Virginia Tech Carilion School of Medicine and North Carolina A\&T State University. This work is funded by the National Science Foundation under grant IIS-1418622.

\section{REFERENCES}

[1] R. Alam, M. Anderson, A. Bankole, and J. Lach. 2018. Inferring physical agitation in dementia using smartwatch and sequential behavior models. In 2018 IEEE EMBS International Conference on Biomedical Health Informatics (BHI). IEEE, 170-173. https://doi.org/10.1109/BHI.2018.8333396

[2] R. Alam, J. Dugan, N. Homdee, N. Gandhi, B. Ghaemmaghami, et al. 2017. BESI: Reliable and heterogeneous sensing and intervention for in-home health applications. In Proceedings of the Second IEEE/ACM International Conference on Connected Health: Applications, Systems and Engineering Technologies (CHASE '17). IEEE Press, Piscataway, NJ, USA, 147-156. https://doi.org/10.1109/CHASE.2017.73 